\title{
¿Dieta sostenible y saludable?. Retrospectiva e implicancias para la nutrición pública
}

\author{
Sustainable and healthy diet?. Retrospective \\ and implications for public health nutrition
}

\begin{abstract}
The objective of this paper was to provide a perspective about a healthy diet, and the challenge posed by sustainability to the consumer as well as to the legislation and the resulting nutrition recommendations. The 21 st Century consumer demands clear cues about foods, particularly those food products that are healthy and promote environmental sustainability. It is in this strain between healthy and sustainable diet that future nutrition recommendations should be framed.

Key words: Sustainable diet, public health nutrition, consumer, nutrition recommendations.
\end{abstract}

\section{INTRODUCCIÓN}

Ya no es novedad para nadie que el planeta en que vivimos se halla bajo un estrés que no había experimentado antes. Los seres humanos, en lugar de ejercer una buena mayordomía de la naturaleza, nos hemos convertido en causantes y cómplices de su destrucción constante. El cambio climático está afectando al planeta tierra donde los desastres naturales ocurren con mayor frecuencia y fuerza que antes. Por lo tanto, es tiempo de tomar conciencia, a nivel colectivo e individual, y actuar de forma consecuente, tomando en cuenta que cualquier acción individual, por ejemplo la elección de alimentos, puede ser el granito de arena con el cual la persona contribuye a la preservación del medio ambiente y a la salud pública en general.

El objetivo de este documento es, primero de dar un breve vistazo al desarrollo de la idea de la sostenibilidad de la dieta, segundo, de identificar algunas complejidades de la adopción de una dieta saludable, y el dilema que constituye para el consumidor la definición de una dieta saludable, y tercero, la provisión de algunos ejemplos en los que se puede lograr tanto sostenibilidad como salud en la alimentación. Para ello, se ha efectuado una puesta al día a través de mini-revisión en la base de datos Medline, con el criterio de búsqueda "sustainable[Title] AND diet[Title]" y se han añadido fuentes identificadas y seleccionadas por el autor.

La sostenibilidad ambiental es una de las prioridades políticas del presente siglo. Por ejemplo, dentro de los desafíos sociales del programa Europeo Horizonte 2020, tanto la sostenibilidad de la agricultura y silvicultura como el medio ambiente se hallan en primera fila, junto con la salud y la seguridad alimentaria (1). En el Reino Unido, el reporte Food
Federico J.A. Pérez-Cueto

Department of Food Science, University of Copenhagen, Dinamarca

Dirigir la correspondencia a: Profesor

Federico J.A. Pérez-Cueto Department of Food Science, University of Copenhagen, Dinamarca Rolighedsvej 26, 1958 Frederiksberg C. Email: apce@food.ku.dk

Este trabajo fue recibido el 04 de Septiembre de 2014 y aceptado para ser publicado el 28 de Junio de 2015.

2030 (2) identifica al cambio climático y a la obesidad como los dos principales desafíos sociales que el país enfrentará, y cuya etiología común los hace objeto de acción dirigida común (3).

Es importante por ello comenzar a entender que la disciplina de la nutrición (y en particular la nutrición pública) no puede aislarse de la conversación, y mucho menos tratar de hallar respuestas fáciles a preguntas complejas. El tema de la obesidad por ejemplo, refleja cómo se interrelacionan la emisión de gases con efecto invernadero, la producción de carne de vacuno, la producción industrial de alimentos y la salud de los individuos (4). Es asimismo un tema económico, ético y político que habrá de ser enfocado desde una perspectiva sistémica, donde la complejidad de interacciones sean tomadas en cuenta.

\section{Retrospectiva}

Haciendo un poco de retrospectiva, la temática que relaciona sostenibilidad y nutrición no es nueva, ya en los 80's se puso sobre el tapete de discusión la necesidad de incluir conceptos de sostenibilidad también en las recomendaciones nutricionales $(5,6)$. Posteriormente, se utilizó el término "econutrición" para llamar la atención hacia el efecto de la pérdida de biodiversidad en la calidad de la alimentación y consecuentemente en la salud humana (7-9). Ya pasado el milenio, se retoma con más fuerza la discusión y los sociólogos de la salud nutricional enfatizan en la integración de la salud, el medio ambiente y la sociedad en la elaboración de políticas alimentarias y nutricionales (10).

Al mismo tiempo, como resultado de modelos matemáticos sobre el efecto de la agricultura en la salud humana, se ha calculado que el sector agrícola contribuye $10-12 \%$ de las 
emisiones de efecto invernadero a nivel mundial, mientras que la desforestación y otros cambios en el uso de la tierra contribuyen con un 6-17\% adicionales de emisiones globales. La producción de alimentos de fuente animal es el mayor contribuyente al efecto invernadero del sector agrícola. Además, debido al crecimiento demográfico, se estima que la demanda global de alimentos de origen animal aumentará sustancialmente en los próximos 30 años, especialmente en economías en transición. Por lo tanto, es imperativo hallar maneras de combinar avances tecnológicos con la reducción en la producción de alimentos de origen animal, de tal forma de contribuir de forma efectiva a lograr las metas nacionales y globales de reducción de gases de efecto invernadero. La principal conclusión de dicho modelo ha sido que la reducción coordinada del consumo de productos de origen animal en poblaciones de elevado consumo, beneficiarían de manera sustancial a la salud pública, por ejemplo mediante una disminución de la enfermedad cardiovascular isquémica (11). Sin embargo, la adopción de una dieta saludable beneficiaría mayormente a países desarrollados, mientras que el coste para los países en crecimiento económico sería elevado (12). Mas aún, debido al crecimiento demográfico y la demanda del mercado, el planeta tendrá que producir mas alimentos, y a menos que se destruya una gran parte de la biodiversidad remanente, esto habrá de lograrse sin ampliar las áreas cultivadas (13). Existen asimismo algunas voces discordantes que sugieren que la situación ambiental no es dramática, y que por lo tanto, no sería necesario promover cambios sustanciales en el consumo de productos de origen animal para lograr sostenibilidad ambiental (14).

\section{La dieta sostenible y el consumidor}

El concepto de dieta sostenible, se inspira de la definición del desarrollo sostenible, que implica un proceso de cambio en el que la explotación de los recursos naturales, el sentido y prioridad de las inversiones, la orientación del desarrollo tecnológico y el cambio institucional están en armonía y mejoran el potencial, presente y futuro, para alcanzar las necesidades y aspiraciones de la humanidad. En consecuencia, se ha definido como dieta sostenible, a "aquella dieta con un impacto medioambiental bajo, que contribuye a la seguridad nutricional y alimentaria, además de ayudar a las generaciones presentes y futuras a llevar una vida saludable. Una dieta sostenible protege y respeta la biodiversidad y los ecosistemas, es culturalmente aceptable, accesible, justa y asequible a nivel económico; adecuada nutricionalmente, segura y saludable, a la vez que optimiza el uso de recursos humanos y naturales" (15).

La propuesta entonces sería de hallar una combinación de alimentos en la dieta que sean a la vez nutritivos, promotores de la salud y sostenibles e inocuos para el medio ambiente. Afortunadamente, en América Latina aun podemos hablar de dietas saludables y sostenibles. En un estudio efectuado a mediados de la primera década del siglo XXI, en el que se documentó la dieta de un grupo de adolescentes del área rural en Bolivia, se vio que en comunidades alejadas se mantienen y cultivan costumbres ancestrales de alimentación (16), las cuales se basan en productos locales y estacionales. De igual forma, una vez efectuados estudios a nivel nacional, en base a los datos de las encuestas de hogares bolivianos, se confirmó el hecho de que las dietas al principio del milenio, si bien se hallaban en transición, eran mayoritariamente en base a productos vegetales y producidos localmente (17). Si bien Latinoamérica está ingresando en etapas mas avanzadas de la transición nutricional (18), la puesta en marcha de acciones preventivas, tendientes a un retorno a la brevedad posible hacia dietas tradicionales, las cuales son mas saludables y sostenibles, lograría frenar los efectos indeseados de dicha transición. Aunque los cambios a lograr fueran aparentemente pequeños, el impacto en la salud de la población sería sustancial, como lo demuestran los estudios sobre la dieta mediterránea: Por cada incremento en el score de dieta mediterránea hay una reducción significativa en mortalidad total $(19,20)$. Esta última observación nos lleva a la siguiente consideración de este documento: Existe un ejemplo de un patrón alimenticio que sea saludable y sostenible a la vez?

La respuesta es si. En 2010, la Fundación Dieta Mediterránea ha generado una nueva pirámide de alimentación saludable la cual integra sostenibilidad ambiental entre sus principios (figura 1). Por primera vez, se cuenta con un ejemplo práctico de que es posible efectuar recomendaciones de alimentación saludable tomando en consideración una serie de parámetros de sostenibilidad, o principios que contribuyen hacia la sostenibilidad ambiental, como ser la biodiversidad, reflejada en una dieta variada, la estacionalidad, traducida en sugerencias de consumo de alimentos de producción local, tradicional y por lo tanto respetuosos del equilibrio ecológico.

Las recomendaciones de alimentación sostenible y saludable, se pueden resumir, entonces, en una dieta rica en productos de origen vegetal, frutas, hortalizas, leguminosas, aceite de oliva virgen y cereales, consumo moderado de alimentos de origen animal, de preferencia variedad de carnes blancas, pescado, mariscos y productos lácteos; consumo limitado e infrecuente de carnes rojas, y eliminación total de la dieta de productos cárnicos preparados y conservados en base a sales de nitrato o nitrito. Estos principios generales, que de alguna forma se basan en sentido común, y ahora reforzados por la evidencia científica, deberán ser aplicados por quienes trabajan en nutrición pública, revalorizando los productos nativos y acorde con las condiciones de cada país y región.

La sostenibilidad como señal para el consumidor

Ahora bien, el consumidor de alimentos, particularmente en los países donde se experimenta crecimiento económico, se ha establecido como un actor preponderante en la demanda de productos que respondan a una serie de señales, ya sean estas de índole cualitativa nutricional o en base a una serie de principios y actitudes respecto a valores que pueden variar desde el bienestar animal, la sostenibilidad ambiental, la ética social (producción de energía con alimentos), hasta el trabajo de niños, o la equidad internacional.

En este maremágnum de señales uno se pregunta cuáles son las principales señales que afectan la decisión del consumidor. Por ejemplo, cuando se trata de pescado, cuyo consumo moderado es sugerido por la mayoría de las políticas nutricionales, debido a su efecto protector ante enfermedades cardiovasculares (22), y a la vez, cuyo consumo tendría un efecto devastador en el stock de pescado a nivel mundial, el consumidor se halla ante la dos señales diferentes. En el caso del Reino Unido, el consumidor británico compra pescado primeramente por razones de salud y no necesariamente por temas de sostenibilidad ambiental (21). La sostenibilidad es una señal corolaria, pero no fundamental en este caso. Si se trata de promoción de pescado originado en una cultura pesquera sostenible, es necesario recurrir a maneras innovadoras para promover y facilitar su consumo.

Otra de las señales que han estado de moda y han marcado la política nutricional en Europa, es la de "bio" u "orgánico". Se trata de una producción que se precia de ser 
amigable con el medio ambiente, con eliminación de los inputs de la producción agropecuaria, principalmente aditivos conservantes, fertilizantes y pesticidas. La producción orgánica es comparativamente más cara que la de tipo convencional, y usualmente dirigida a un mercado elitista. Sin embargo, existe la creencia generalizada de que el producto orgánico o bio, es de mejor calidad nutricional, y por lo tanto mas saludable, pese a que la evidencia científica no la sostenga (23-26). Pero se ha enraizado de tal forma que, en un estudio reciente en Dinamarca, se pudo observar que quienes consumen orgánico, lo hacen porque llevan ya un estilo de vida saludable, y por lo tanto, el consumo de dichos productos no es motivado por el deseo de ser mas saludables, sino una consecuencia de un estilo de vida (26). El mensaje implícito es que hay un grupo creciente de consumidores para quienes la sostenibilidad ambiental se ha convertido en una señal necesaria el momento de adquirir sus alimentos $(27,28)$, mientras que para otros segmentos de consumidores, la sostenibilidad ambiental pasa a segundo plano el momento de efectuar decisiones alimenticias.

Tierra y trabajo en servicio del capital?

Originalmente, el desarrollo económico de las naciones tenía como objetivo primordial mejorar las condiciones de vida y de salud de los seres humanos, es decir el capital humano, la fuerza laboral. Para ello, estaban al servicio de este principio fundamental, tanto la tecnología agrícola y el manejo responsable de la naturaleza, como el capital financiero. Hemos llegado a un punto en la historia de la humanidad en el cual este orden está de cabeza, y ahora los factores de producción, tierra y trabajo humano se hallan supeditados al crecimiento del capital. Esta mentalidad requiere un cambio, sobre todo si es que tenemos la intención de que las generaciones futuras puedan vivir sobre el planeta tierra. Se requiere un cambio de perspectiva: debemos auto-percibirnos como administradores

\section{Pirámide de la Dieta Mediterránea: un estilo de vida actual}

Guía para la población adulta
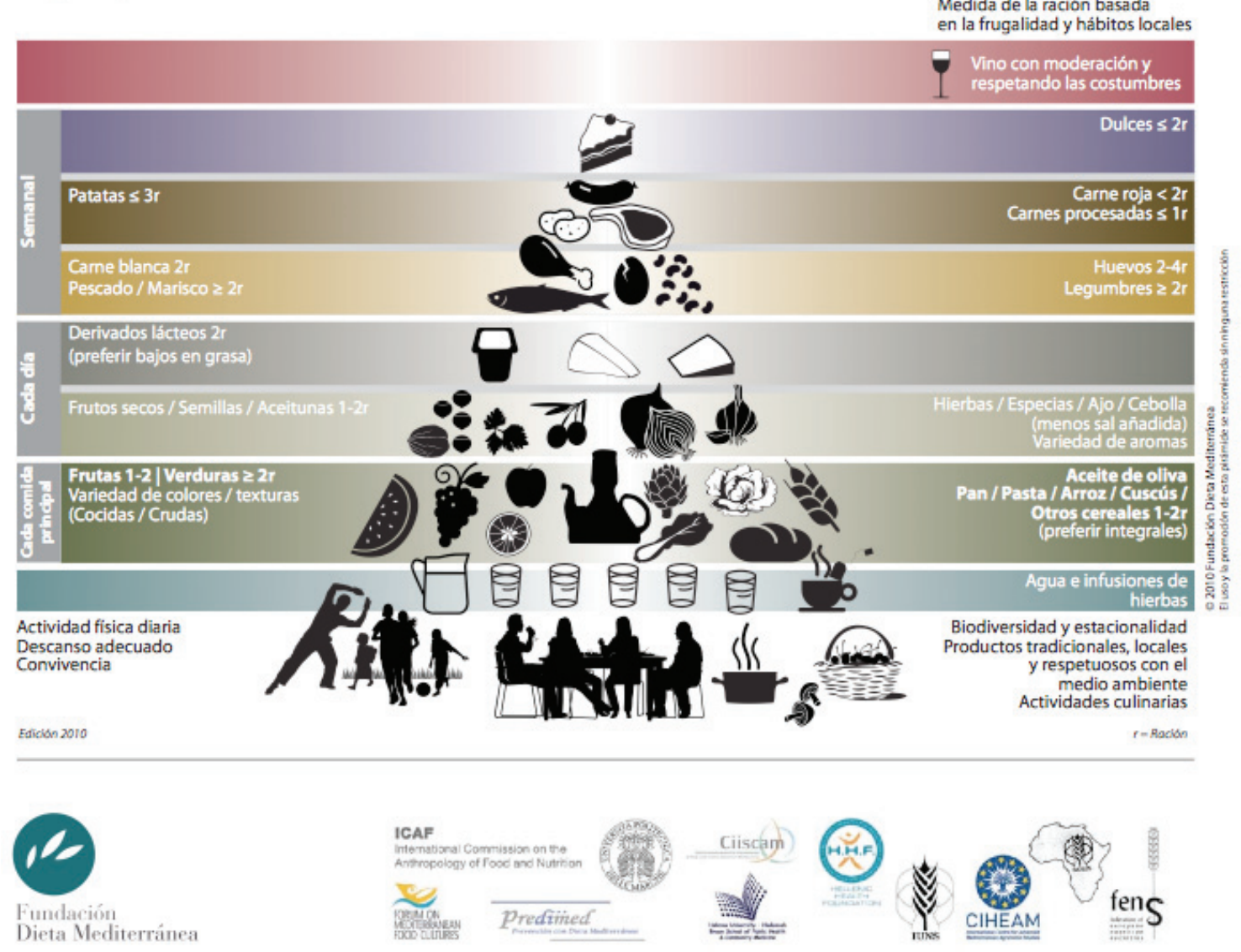

Usada con permiso, disponible en http://dietamediterranea.com/dietamed/piramidedietamediterranea_descarga.pdf "r" significa ración. 
$\&$ mayordomos del planeta, y no sus destructores. Tenemos responsabilidades profesionales, como nutricionistas, de fomentar dietas equilibradas y saludables, donde el objetivo primordial sea el de garantizar una mejor calidad de vida para la población, y al mismo tiempo, de garantizar la sobrevivencia de la raza humana.

\section{Implicancias para la nutrición pública}

Quienes trabajamos en nutrición pública, por lo tanto, tenemos la responsabilidad de llamar a cuentas a quienes dirigen las naciones, regiones y municipios, debemos llegar a ellos con la evidencia que la ciencia nos permite, y abogar por una distribución democrática y justa de los alimentos en el sistema global, y promover, por el bienestar general y la salud pública el concepto de consumo suficiente, que es intuitivamente contrario al consumo desmesurado que promueve la economía de mercado libre.

Por otro lado, es crucial el rol de consejeros para el sector privado. El nutricionista público es el interlocutor natural, y quien habrá de revelar las necesidades de acción por parte de la industria, ya sea reformulando productos, innovando en tecnología, y deberá ser el intérprete de las recomendaciones y legislaciones sobre alimentación saludable tanto para el público en general, como para el sector productivo. Finalmente, en adelante, para quienes trabajan en nutrición pública, será primordial tomar una actitud militante en favor del medio ambiente al momento de generar nuevas recomendaciones, y de proporcionar consejo profesional a legisladores y otros actores sociales.

\section{RESUMEN}

El objetivo de esta comunicación fue proporcionar una perspectiva sobre aspectos de la dieta saludable y el desafío que conlleva la sostenibilidad de la dieta, tanto para el consumidor como para la legislación y para las recomendaciones nutricionales. El consumidor del Siglo XXI demanda señales claras sobre los alimentos, particularmente que éstos sean saludables y fomenten la sostenibilidad ambiental. Es en esta tensión que las recomendaciones nutricionales deberán enmarcarse en el futuro.

Palabras clave: Dieta sostenible, nutrición pública, consumidor, recomendaciones nutricionales.

\section{REFERENCIAS}

1. Comisión Europea, acceso en Marzo 2014 http:// ec.europa.eu/programmes/horizon2020/en/h2020section/societal-challenges

2. Department for Environment, Food and Rural Affairs (2010). Food 2030. London: HM Government. https://www. london.gov.uk/sites/default/files/food2030strategy.pdf

3. Macdiarmid JI. Is a healthy diet an environmentally sustainable diet? Proc Nutr Soc. 2013;72(1):13-20.

4. Friel $S$, Dangour AD, Garnett $T$, Lock $K$, Chalabi Z, Roberts I, Butler A, Butler CD, Waage J, McMichael AJ, Haines A. Public health benefits of strategies to reduce greenhouse-gas emissions: food and agricultura, Lancet 2009;374(9706):2016-25.

5. Gussow JD, Clancy $K$. Dietary guidelines for sustainability. J Nutr Educ 1986;18:1-4.

6. Gussow JD. Dietary guidelines for sustainability: twelve years later, J Nutr Educ. 1999;31:194-200.

7. Wahlqvist ML. Connected Community and Household Food-Based Strategy (CCH-FBS): its importance for health, food safety, sustainability and security in diverse localities. Ecol Food Nutr. 2009;48(6):457-81.

8. Wahlqvist ML. Chronic disease prevention: A life-cycle approach which takes account of the environmental impact and opportunities of food, nutrition and public health policies - the rationale for an eco-nutritional disease nomenclature, Asia Pac J Clin Nutr. 2002;11 (Suppl 9):S759-62.

9. Wahlqvist ML, Specht RL. Food variety and biodiversity: Econutrition, Asia Pac J Clin Nutr. 1998;7(3/4):314-9.

10. Barling, D., Lang, T. and Caraher, M. Food Policy: integrating health, environment and society. Oxford: Oxford University Press. 2009.

11. Lock K, Smith RD, Dangour AD, Keogh-Brown M, Pigatto $G$, Hawkes C, Fisberg RM, Chalabi Z. Health, agricultural, and economic effects of adoption of healthy diet recommendations, Lancet 2010;376(9753):1699-709.

12. Friel S, Dangour AD, Garnett T, Lock K, Chalabi Z, Roberts I, Butler A, Butler CD, Waage J, McMichael AJ, Haines A. Public health benefits of strategies to reduce greenhouse-gas emissions: food and agricultura. Lancet 2009;374(9706):2016-25.

13. Hawkesworth $S$, Dangour AD, Johnston D, Lock K, Poole N, Rushton J, Uauy R, Waage J. Feeding the world healthily: the challenge of measuring the effects of agriculture on health. Philos Trans $R$ Soc Lond B Biol Sci. 2010;365(1554):3083-97.

14. Macdiarmid JI, Kyle J, Horgan GW, Loe J, Fyfe C, Johnstone A, McNeill G. Sustainable diets for the future: Can we contribute to reducing greenhouse gas emissions by eating a healthy diet? Am J Clin Nutr. 2012;96(3):632-9.

15. FAO 2012: http://www.fao.org/docrep/016/i3004e/i3004e. pdf

16. Pérez-Cueto FJ, Almanza-López MJ, Pérez-Cueto JD, Eulert ME. [Nutritional status and diet characteristics of a group of adolescents from the rural locality Calama, Bolivia], Nutr Hosp. 2009;24(1):46-50.

17. Pérez-Cueto FJ, Naska A, Monterrey J, Almanza-Lopez M, Trichopoulou A, Kolsteren P. Monitoring food and nutrient availability in a nationally representative sample of Bolivian households, Br J Nutr. 2006;95(3):555-67.

18. Barría RM, Amigo H. Transición Nutricional: una revisión del perfil latinoamericano, Arch Latinoam Nutr. 2006;56(1):3-11.

19. Estruch R, Ros E, Salas-Salvadó J, Covas MI, Corella D, Arós F, Gómez-Gracia E, Ruiz-Gutiérrez V, Fiol M, Lapetra J, Lamuela-Raventos RM, Serra-Majem L, Pintó X, Basora J, Muñoz MA, Sorlí JV, Martínez JA, Martínez-González MA; PREDIMED Study Investigators. Primary prevention of cardiovascular disease with a Mediterranean diet. $N$ Engl J Med. 2013;368(14):1279-90.

20. Guasch-Ferré $M$, Bulló $M$, Martínez-González MÁ, Ros E, Corella D, Estruch R, Fitó $M$, Arós F, Wärnberg J, Fiol $M$, Lapetra J, Vinyoles E, Lamuela-Raventós RM, Serra-Majem L, Pintó X, Ruiz-Gutiérrez V, Basora J, Salas-Salvadó J; PREDIMED study group. Frequency of nut consumption and mortality risk in the PREDIMED nutrition intervention trial, BMC Med 2013 Jul 16;11:164. doi:10.1186/17417015-11-164.

21. Clonan A, Holdsworth $M$, Swift JA, Leibovici D, Wilson $P$. The dilemma of healthy eating and environmental sustainability: the case of fish, Public Health Nutr. 2012;15(2):277-84.

22. Perez-Cueto F, Pieniak Z, Verbeke W. Attitudinal determinants of fish consumption in Spain and Poland. Nutr 
Hosp. 2011;26(6):1412-9.

23. Dangour AD, Lock K, Hayter A, Aikenhead A, Allen E, Uauy R. Nutrition-related health effects of organic foods: a systematic review, Am J Clin Nutr. 2010;92(1):203-10.

24. Dangour AD, Allen E, Lock K, Uauy R. Nutritional composition \& health benefits of organic foods - using systematic reviews to question the available evidence, Indian J Med Res. 2010;131:478-80.

25. Dangour AD, Dodhia SK, Hayter A, Allen E, Lock K, Uauy $R$. Nutritional quality of organic foods: a systematic review, Am J Clin Nutr. 2009;90(3):680-5.
26. He C, Breiting S, Perez-Cueto FJ. Effect of organic school meals to promote healthy diet in 11-13 year old children. A mixed methods study in four Danish public schools. Appetite 2012;59(3):866-76.

27. Hoogland CT, de Boer J, Boersema JJ. Food and sustainability: do consumers recognize, understand and value on-package information on production standards? Appetite. 2007;49(1):47-57.

28. Schleenbecker R, Hamm U. Consumers' perception of organic product characteristics. A review, Appetite 2013;71:420-9. 